\title{
Investigation of the Protonated State of HIV-1 Protease Active Site
}

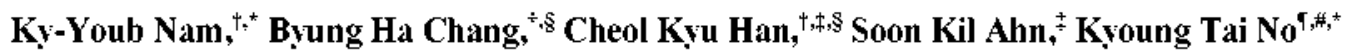 \\ Deparment of Chemistry and CAMD Research Center. Soongsil Lminersin, Seoul 156-743. Korea \\ "Chong Kun Dang Research Institute, 15-20. P.O. Box 74, Cheon An 330-830, Korea \\ 'Department of Biotechnologv, Yonsei Cinversin, Seoul 120-749, Korea \\ Received February 27, 2003
}

\begin{abstract}
We have performed ab initio calculation on the active site of HIV-I protease. The FEP method was used to determine the binding free energy of four different of protonated states of HIV-I protease with inhibitor. The stnicture of the active site and hole stnicture was taken from the X-ray cry stallographic coordinates of the $\mathrm{C}_{2}$ sy'mmetric inhibitor A74704 protease bound. The active site was modeled with the fragment molecules of binding pocket. acetic acid/ acetate anion (Asp25. Asp125), formamide (amide bond of Thr26/Gly 27. Thrl26/ Gly 127), and methanol as inhibitor fragment. All possibly protonated states of the active site were considered. which were diprotonated state $(0,0)$. monoprotonated $(-1,0),(0,-1)$ and diunprotonated state $(-1,-1)$. Once the binding energy $\Delta \mathrm{E}_{\text {bun }}$ of each model was calculated. more probabilistic protonated states can be proposed from binding energy. From $a b$-initio results. the FEP simulations were performed for the three following mutations: I) Asp25 $\cdots$ Asp125 $\rightarrow$ AspH25 $\cdots$ Aspl25, ii) Asp25 $\cdots$ Aspl25 $\rightarrow$ Asp25 $\cdots$ AspH125, iii) AspH25 $\cdots$ Aspl $25 \rightarrow$ AspH25 $\cdots$ AspH 125. The free energy difference between the four states gives the information of the more realistic protonated state of active site aspartic acid. These results provide a theoretical prediction of the protonation state of the catalytic aspartic residues for A74707 complex, and may be useful for the evaluation of potential therapeutic targets.
\end{abstract}

Key Words : HIV-I protease. Protonated state, ab initio. SCI-PCM SCRF. Free energy perturbation

\section{Introduction}

Human Immunodeficiency Virus Type-1 (HIV-1), the cause of acquired immune deficiency syndrome (AIDS). is a member of a retrovinus family. 'HIV-1 Protease (PR), a member of the aspartic protease family of enzymes. processes polypeptides Pr160gag-pol and Pr55gag transcribed form the gag and pol genes late in the viral replicative cycle: This macromolecule processes HIV polyproteins to produce structural and functional proteins. including the protease itself, and has shown itself to be essential for viral replication. ${ }^{3}$ HIV-1 protease has become an important target for the design of antiviral agents for AIDS. HIV-1 protease is a homo-dimer. which is composed of two identically folded 99 amino acids subunits to form a hỵdrophobic binding pocket and the active site formed with two aspartyl residues.

The crystallographic structures of both recombinant and synthetic HIV-1 protease have been reported. ${ }^{48}$ The cellular protease contain an extended $\beta$-hairpin loop. which rolls a flap that embraces the substrate in a tightly bound formation to the active site cavity. The flap undergoes a major structural rearrangement during the complex to make farorable von der Wods contacts and hydrogen bonding orientations with

${ }^{*}$ To whom all correspondence should be addressed. Tel $+82-2$ 825-1785: Fax: +82-2-825-1795; E-mail: knnäbmdrcorg: ktho $t$ bmdre org

Member of Hyperstructured Organic Material Research Center, Korea

sCurrent address: IDR Tech. Inc. B-I, Kwacheon Officetel 5th Fl., Byolyang-Dong, Kwacheon-shi, Kyonggi-Do 427-040, Korea the substrates.

The active site formed with two catalytic aspartic acid residues. Asp-25 and Asp-125, has $C_{2}$ point symmetry in neutral state. Those two aspartyl residues are located at the dimeric interface and are a different structural feature. indistinguishable from the other of monomeric aspartic protease. A conserved water molecule. $\mathrm{H}_{2} \mathrm{O} 301$. is located between the flaps and the substrate or peptidomimetic analog inhibitors and engages in lydrogen-bond networking involving the ligand and the amide nitrogen of Ile50/Ilel50 in the $\beta$-hairpins. Overall, the peptide-like analogs bind to HIV-1 protease in the extended conformation. with their amide groups involved in conserved lydrogen bonds with the active site residues and $\mathrm{H}_{2} \mathrm{O}(301)$. Two side chains of the inhibitor occupy hydrophobic sites that alternate above and below the backbone chain. which are designated according to the convention of Schechter and Berger (P1. P2. ... S1. $S 2, \ldots)^{10}$ Since the structure of the HIV-l protease was determined by X-ray crystallographic and NMR experiments. it has been a good target for structure-based drug designs for AIDS therapeutics. For the inhibitor design, the structure of the active site must be well-defined. not only as a 3dimensional shape. but also as a chemical state. In the HIV-1 protease. the determination of the protonated states of the Asp-25 and Asp-125 is crucial for high affinity inhibitor design. For illumination of the protonated states of HIV-1 protease with several bound inhibitors binding and at various $\mathrm{pH}$-conditions, several works have performed. ${ }^{11-16}$

Meek and co-workers have suggested the general acidgeneral base mechanism and protonated state for HIV-1 
protease in which the scissile nitrogen is protonated at an intermediate step. on the basis of a variety of experimental studies. ${ }^{11-13}$ They explored kinetic study for un-protonated and protonated active site aspartyl groups. Asp-25 and Asp125. HIV-1 protease was examined over a $\mathrm{pH}$ range of $3-7$ with two competitive charged inhibitors. Two aspartyl groups that have $\mathrm{p} K$ value $4.9 \pm 0.06$ and $3.1 \pm 0.1$ must be deprotonated in the case of a cationic inhibitor. One of two aspartyl groups with an uncharged inlibitor was deprotonated $\left(\mathrm{p} K_{1}=3.3 \pm 0.1\right)$. and the other was protonated $\left(\mathrm{p} K_{2}=\right.$ $5.3 \pm 0.09)$

Beveridge and co-worker theoretically deduced the protonated state of two different HIV-1 protease-inhibitor complexes. U85548E and MVT-101, using molecular dynamic (MD) simulation. From MD studies. they suggested that U85548E. hydroxyethylene moiety contains no ionizable groups to neutralize a resultant charge. and that MVT-101. a secondary amine as peptide isostere containing a positive charge. the di-anion model in the four state for MVT-101 and diprotonate state in the four state for U85548E accurately reproduces and agrees with the X-ray crystal structures. ${ }^{14}$

Chen and Tropsha reported binding affinities of the $S$ and $\mathrm{R}$ isomer of U85548E by the free energy perturbation method where they calculated the difference in free energy between a monoprotonated state and two diprotonated states. ${ }^{15}$ They suggested that U85548E binds to a monoprotonated state of HIV protease and that the $\mathrm{S}$ inhibitors bind stronger than the $\mathrm{R}$ inhibitor by about $2.8 \mathrm{kcal} / \mathrm{mol}$.

Ferguson and Kollman detemined the relative binding free energy of the $\mathrm{S}$ and $\mathrm{R}$ isomers of JG365 to the HIV-1 protease at various protonated states by the free energy perturbation method. ${ }^{16}$ Three possible protonated states were considered. including mutated forms of the $\mathrm{S}$ and $\mathrm{R}$ isomers. and the binding free energy difference was compared with experimental free energy. which resulted in similar computational results mono-protonated state of Asp 125.

In this work several protonated states of HIV-l protease with inhibitor A74704, will be investigated. In order to illuminate the physically realistic protonated states of the HIV-1 protease and its complex with the inhibitors both quantum chemical and the free energy simulation approaches were introduced.

\section{Methods}

A. Model compounds. In order to investigate the relative stability between different protonated states of the HIV-1 protease when it is complexed with the inhibitor. A74704ab initio $\mathrm{MO}$ calculations were performed. All the possible protonated states of the active site were taken into account as models. The HIV-1 protease. without inhibitor has $\mathrm{C}_{2}$ symmetry axis. The HIV-l protease of the aspartic oxygen atom. one aspartic amino acid is closer to the $C_{2}$ axis than the outer one. This oxygen is designated as inner oxygen and another is designated as outer oxygen. The models can be classified into three classes: class I. Model I-Model IV. has one protonated aspartic. Model I. protonated on the inner

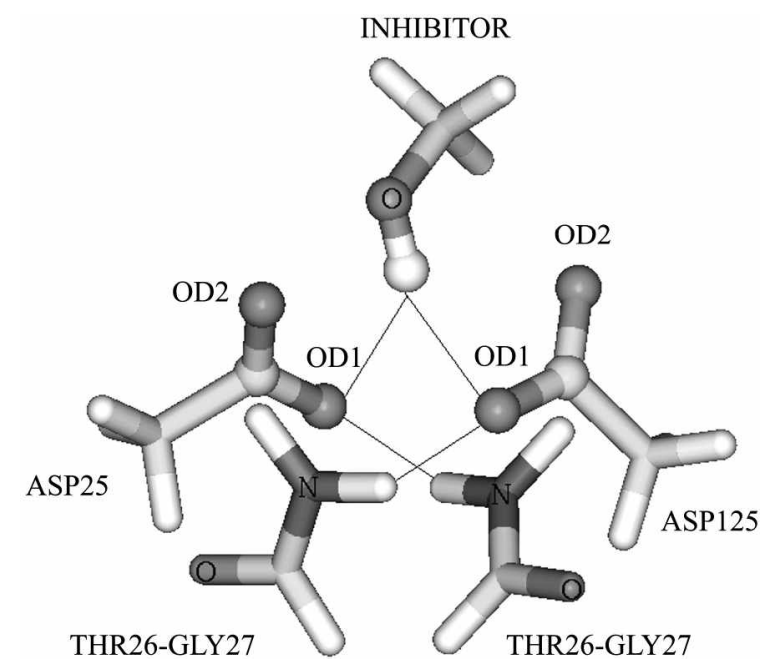

Figure 1. Illustrate the active site of $\mathrm{HTV}-1$ protease-A 74704 complex. The active site consists of the two carboxyl groups from Asp25 and 125 . Protons involved in tom hydrogen bonds between the inhibitor and aspartic residues. Nitrogens of Gly 27 and Gly 127 form hydrogen bonds to active site of Asp25 and Aspl 25

oxygen OD1 of Asp25: Model II. protonated on the inner oxygen ODl of Asp 125: Model III, protonated on the outer oxygen OD2 of Asp25: Model IV, protonated on the outer oxygen OD2 of Asp125: class $I$. Model V. both aspartic acids are deprotonated: class $I I$. Model VI-Model IX. neutral models in which both aspartic acids are protonated: Model VI. protonated on OD1 of Asp25 and on OD2 of Asp125: Model VII. protonated on OD2 of Asp25 and on ODl of Asp 125. Model VIII. protonated on OD2 oxygen of both Asp25 and Asp125: Model LX. protonated on ODl of both Asp25 and Asp 125. The proposed models of the HIV-1 protease were simplified for the $a b$ initio MO calculations. In the simplified models. aspartate and aspartic acid were replaced with acetic and acetic acid respectively: The peptide bonds formed between Thr26 and Gly27/Thr126 and Gly 127 were replaced with two formamides. The hydrogen of the peptide bond forms hydrogen bonds with aspartate/ aspartic acid and $\mathrm{OH}$ of the inhibitor. Figure 1 shows simple model structures. The ball and stick regions represent the optimized geometries, and the stick line represents the fixed geometries during geometry optimizations.

B. $A b$ initio calculations. We performed $a b$ initio SCF calculation using Gaussian $94 .{ }^{17}$ All geometry optimizations were performed with the $6-31+\mathrm{G}^{* *}$ basis set and the relative stability of models were calculated. Both fonmanides bonds and all the methyl groups in the model were fixed during optimization procedures. Models have different proton numbers each other due to the different protonated states. In the gas phase. there is no problem in comparing the stability between different protonated states if the deprotonated proton is removed or far apart from the model. as the energy of the proton is zero in quantum chemical calculations in the gas phase. However. in solution, for comparing the stability between different protonated states. one must clearly define the state of the proton or protons. i.e.. is the proton bonded to 
water. or bonded to another part of the protein. forming an $\mathrm{X}-\mathrm{H}$ bond. Therefore it is assumed that the deprotonated proton from the $-\mathrm{COOH}$ forms an $\mathrm{O}-\mathrm{H}$ bond somewhere in the protein

The relative energy of a model A with respect to a reference model in the phase $\mathrm{X}$ was calculated as follows.

$$
\Delta E_{r e J, Y}^{\dagger}=\left(E_{X^{\prime}}^{\dagger}-E_{X^{\prime}}^{r e f}\right)-\Delta n \Delta E_{O H . x}
$$

$E_{X}^{\dagger}$ and $E_{X}^{r e f}$ are the $\mathrm{HF}$ energies of model A and reference model is model III. respectively. in phase $X . \Delta n$ is the difference in the number of protons. $n^{i}-n^{r f}, n^{\text {ref }}=1 . \Delta n$ can be $+1,0,-1 . \Delta E_{O H . . .}$ is obtained as follows.

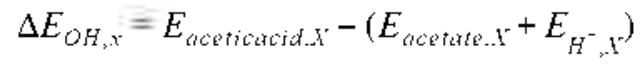

The binding energy between model protease (two acetic acids and two formamides in the simplified models) and the inhibitor (methanol in the simplified model) was calculated as follows.

$$
E_{\text {binding }}=E_{\text {model }}-\left(E_{\text {proterse }}+E_{\text {inhibiror }}\right)
$$

$E_{p r t a t a s s}$ and $E_{i z h t i b i t o r}$ were calculated with optimized geometry using same basis set $6-31+\mathrm{G}^{* *}$. As in Figure 1, the model protease was represented simply by two acetic acid/anion and two formanides.

To calculate the relative stability between simplified models in dielectric medium. self-consistent reaction fields (SCRF) calculations were performed with a self-consistent isodensity polarizable continuum model (SCI-PCM) ${ }^{18.19} \varepsilon=$ 4.0 was used as dielectric constant to a mimic protein core environment. because two aspartic acids are located in the HIV-l protease core region.
(1)

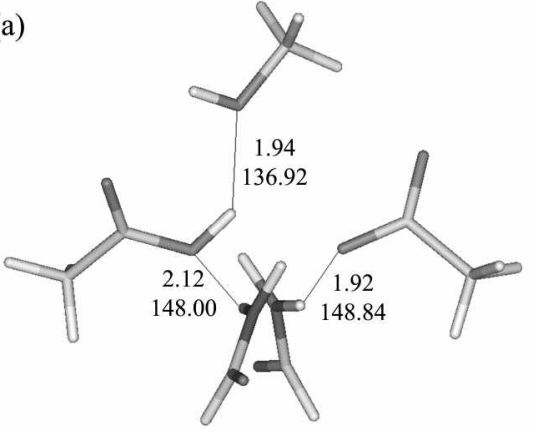

(d)

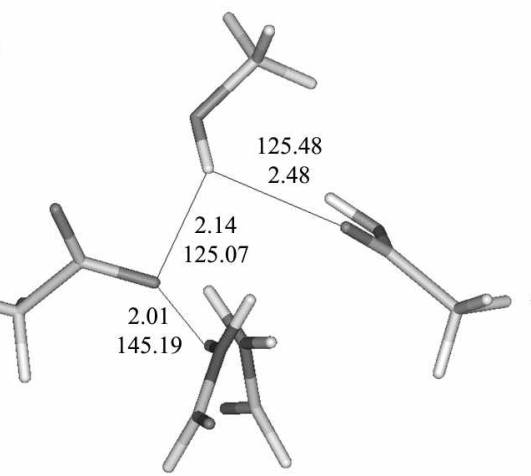

(g)

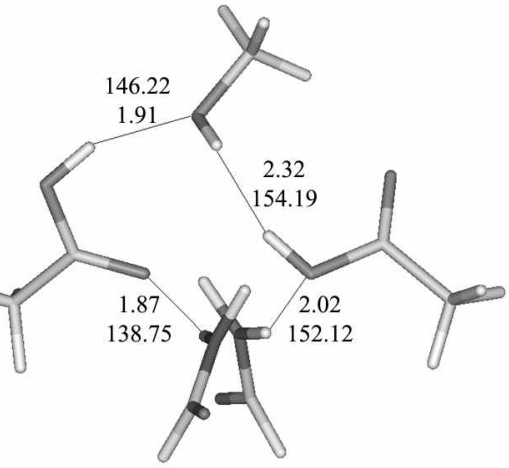

(b)

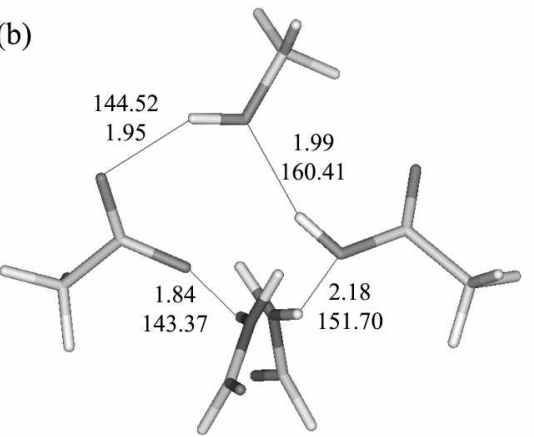

(e)

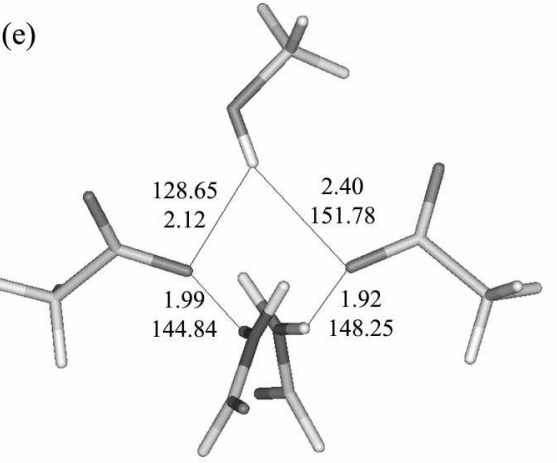

(h)

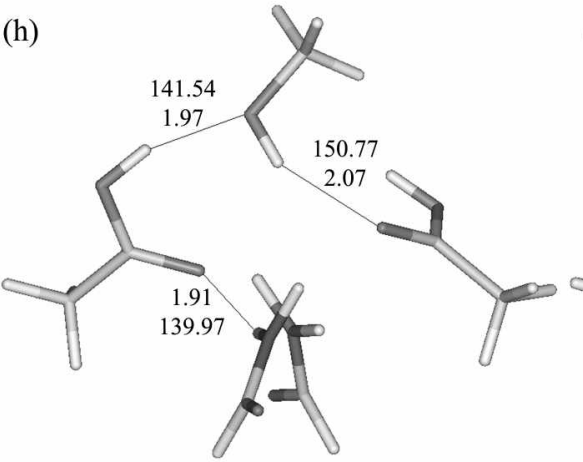

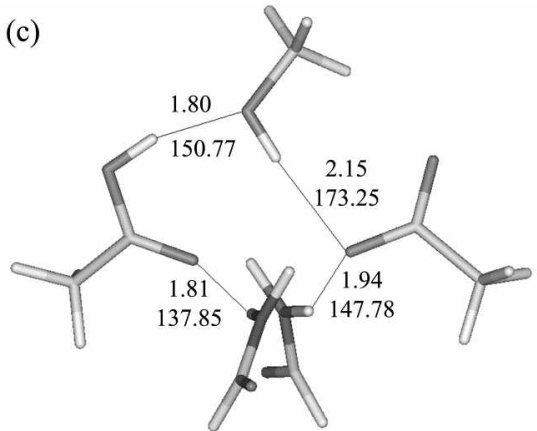

(f)

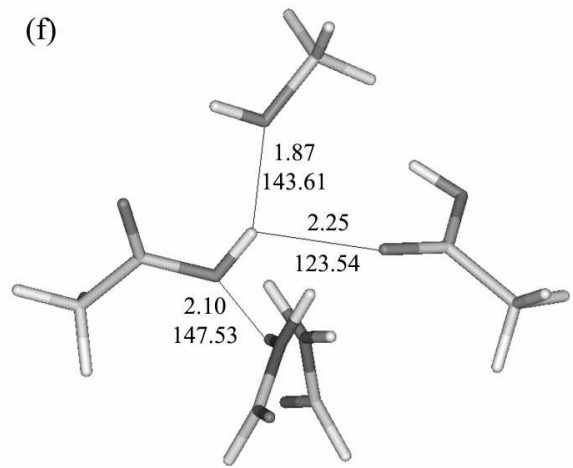

(i)

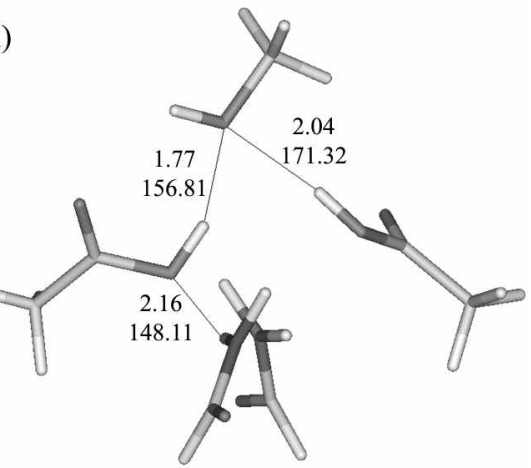

Figure 2. Optimized geometries ( $\mathrm{HF} / 6-31+\mathrm{G}^{* *}$ basis set) for the active site of HIV-l protease-A 74704 complex. Direction hy droxyl group in inhibitor are difterent each state for hydrogen bond: chemical structures and structural models of the monoprotonated (a-d), dianion (e), and diprotonated (t-i) fonns. 
C. Calculation of free energy difference. The free energy perturbation method was used for computing the relative free energy of a certain protonated state by constnucting a nonphysical path which connects the desired initial and terminal states. This approach directly enables the calculation of relative change in the free energy difference between two protonated states by computationally simulating the mutation of one protonated state to the other. The total free energy change for the mutation from the initial to the final state is computed by summing incremental free energy changes over several windows visited by the state variable changing from 0 to 1 . The thermody namic integration of the relative free energy assumes that the free energy change can be expressed as an integral.

$$
\Delta A=\int_{0}^{1} \frac{\delta A(\lambda)}{\delta \lambda} d \lambda
$$

The above equation can be integrated numerically. FDTI (finite difference themodynamic integration) ${ }^{20}$ in the Discover ${ }^{2}$ program employs the perturbation formalism to numerically compute the derivatives of the free energy function. with respect to the coupling parameter $\lambda$.

$$
\begin{aligned}
\Delta A_{i} & =A\left(\lambda_{j}\right)-A\left(\lambda_{j} \pm \delta \lambda\right) \\
& =-k_{b} T \ln \left(\exp \left[-\left(E\left(\lambda_{j}\right)-E\left(\lambda_{i} \pm \delta \lambda\right)\right) / k_{b} T\right]_{i}\right)
\end{aligned}
$$

Conputing $\Delta A_{i}$ for six values of $\lambda$ spanning the integral from 0.0 to 1.0 . Total free energy change $\Delta A$ can be estimated.

$$
\Delta A=-k_{b} T \sum_{i=1}^{k} \frac{\ln \left(\exp \left[-\left(E\left(\lambda_{i}\right)-E\left(\lambda_{i} \pm \delta \lambda\right)\right) / k_{b} T\right]\right)}{\delta \lambda} \Delta \lambda_{i}
$$

Starting structures were built by adding hydrogens to the heavy atom positions of an HIV-1 protease-inhibitor crystal structure (9hyp) in the Brookhasen protein data bank (PDB) using CVFF. ${ }^{21-23}$ The relatively stable protonated states were introduced as the states in the free energy perturbation simulation. The relative stability was obtained from the $a b$ initio calculations. For the construction of the thermodynamic cycle. Figure 3. unprotonated states. Model V. two monoprotonated states. Model II and Model III. and diprotonated state Model VIII were introduced.

Since the HIV-1 protease is too large to use the periodic boundary condition for solution simulation. in this simulation. the HIV-1 protease complexes were hydrated around OD2 of Asp 25 by 27 A diameter water sphere of 937 individual water molecules. A switching function was used for long range nonbonding energy terms with a cut-off value $12 \mathrm{~A}$. Before the molecular dynamic simulation, the HIV-1 protease complexes were relaxed by 5000 steps of conjugate gradient minimization method to eliminate the initial strain in the molecule.

Numerical integration by the leap frog integrator with a 1 fis step size was used for molecular dynamic calculation. The molecular dynamics calculations for the solvated system were performed on nuning at equilibrium for $30 \mathrm{ps}$. follow-

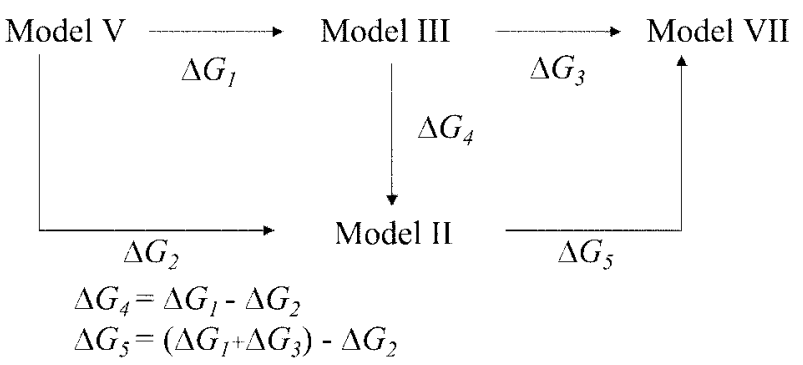

Figure 3. The scheme of the thermodynamic oycle used in this study $\Delta G_{1}, \Delta G_{2}$, and $\Delta G_{3}$ represent the free energy differences of the protonated state that can be computed by the free energy perturbation.

ed by $70 p s$ at $300 \mathrm{~K}$. The free energy differences were collected at every 10 steps. and perturbation was used for $6 \lambda$ points.

\section{Results and Discussion}

Both relative and binding energies of the models were calculated with $6-31+\mathrm{G}^{* *} \mathrm{HF} a b$ initio $\mathrm{MO}$ calculations and are summarized in Table 1. The model III, lowest relative energy and strongest complex form. was used as a reference. To include the polarization effects of the hydrogen bonding in the models. $2 \mathrm{p}$ and $3 \mathrm{~d}$ orbital are introduced for the hydrogen and oxygen/nitrogen, respectively: In the gas phase. the monoprotonated states. models I-IV. are relatively' more stable phases than di- and unprotonated states under the assumption that is introduced to define $\Delta E_{\text {Fel }}$. For monoprotonated states. the relative energies were obtained in the order. Model III > Model II > Model I > Model IV. Diprotonated states have higher relative energy. about $35.0 \mathrm{kcal} / \mathrm{mol}$.

Table 1. Relative energy ( $\Delta E_{\text {rel.gas }}^{-i}$ ). Binding energy ( $E_{\text {binding }}$ ) and Number of hydrogen bond for $\mathrm{HIV}$-I protease-inhibitor complex in gas phase

\begin{tabular}{lccc}
\hline & $\begin{array}{c}\text { Relative energy Binding energy } \\
\Delta E_{r e l, k s s}^{-1}\end{array}$ & $\begin{array}{c}\text { Number of } \mathrm{HB}^{a} \\
E_{t y n i m s}\end{array}$ & $\begin{array}{c}\text { (strong, intenmediate, } \\
\text { Heak) }\end{array}$ \\
\hline Model I & 3.31 & -0.95 & $(0,0,3)$ \\
Model II & 2.40 & -2.91 & $(0,1,3)$ \\
Model III & 0.0 & -18.18 & $(0,2,2)$ \\
Model IV & 22.23 & 2.80 & $(0,0,2)$ \\
Model V & 62.06 & -2.76 & $(0,0,3)$ \\
Model VI & 38.64 & 0.67 & $(0,0,3)$ \\
Model VII & 39.54 & 3.02 & $(0,0,3)$ \\
Model VIII & 34.61 & -1.93 & $(0,1,2)$ \\
Model IX & 37.25 & -11.10 & $(0,0,3)$ \\
\hline
\end{tabular}

In counting the numbers of HBs according to their strength. they were classified into three classes.

Strong: Hydrogen bond length. $\quad \cdots .75 \AA$

Intermediate: Hydrogen bond length. $1.76 \AA 1.85 \AA$

Weak: $\quad$ Hydrogen bond length. $1.86 \AA-2.20 \AA$

Other hydrogen bonding atomic pairs which can not satisfy the above three condition were not introduced in the counting.

The hydrogen bond classifications are not general and applicable 6$31-G^{* * *}$ basis set. 
Table 2. Relative energies ${ }^{a}\left(\Delta E_{r e l+1}^{-1}, \Delta E_{r e l-83}^{4}\right.$ ) at two dielectric continuum medium $(\varepsilon=4.0$, protein core environment: $\varepsilon=78.3$, aqueous solution enviroment) for $\mathrm{HIV}-1$ protease complexes

\begin{tabular}{lcc}
\hline & $\begin{array}{c}\text { Relative energy } \\
\Delta E_{r e l .4}^{-t}\end{array}$ & $\begin{array}{c}\text { Relative energy } \\
\Delta E_{r \text { rl, } ; 8.3}^{t}\end{array}$ \\
\hline Model I & 1.98 & 0.82 \\
Model II & 1.21 & 0.46 \\
Model III & 0.00 & 0.00 \\
Model IV & 16.49 & 10.94 \\
Model V & 32.24 & 20.95 \\
Model VI & 12.53 & 2.29 \\
Model VII & 13.84 & 3.65 \\
Model VII & 9.45 & -0.42 \\
Model IX & 11.31 & 1.24 \\
\hline
\end{tabular}

"HF:6-31+G** SCI-PCM calculations (kcal: mol)

than Model III. Unprotonated state. Model V. is quite unstable. $62.06 \mathrm{kcal} / \mathrm{mol}$. more than Model III. The structure of Model III. with ionized Aspl25 and protonated Asp25 oxygen OD2 2 s is more stable than Model II by $3.39 \mathrm{kcal} /$ mol. The binding energy of Model III can be readily understood by comparing the optimized structures. Four hydrogen bonds were formed in the Model III inhibitor complex. Two hy'drogen bonds are formed between the inhibitor and Model III: i) the inhibitor oxy'gen and the proton on $\mathrm{OD}_{35}\left(\mathrm{R}_{\odot \mathrm{H}}=\right.$ $\left.1.80 \mathrm{~A}: \angle \mathrm{OD} 2_{3}-\mathrm{H} \cdots \mathrm{O}_{\text {inhibitor }}=150.77^{\circ}\right)$ and ii) the proton on the iuhibitor-OH and $\mathrm{ODI}_{1 \unlhd}\left(\mathrm{R}_{\mathrm{OH}}=2.15 \AA ; \angle \mathrm{O}_{\text {inthibitor }}-\right.$ $\left.\mathrm{H} \cdots \mathrm{ODl}_{1 \unlhd s}=173.25^{\circ}\right)$. There are two intra HIV-I protease hydrogen bonds; i) the amide proton in Gly 27 and $\mathrm{ODl}_{\text {s. }}$

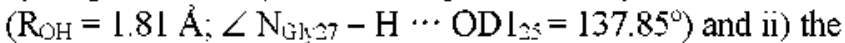
anide proton in Gly 127 and $\mathrm{ODl}_{1 \leq 5}\left(\mathrm{R}_{O H}=1.94 \mathrm{~A}_{;} \angle \mathrm{N}_{\text {Gly } 127}\right.$ $\left.-\mathrm{H} \cdots \mathrm{ODI}_{1 s}=147.78^{\circ}\right)$. Since all the four hydrogen bonds have suitable bond distances and bond angles for hydrogen bond formation, the Model III can form a strong coniplex with the inhibitor. Our previous conputational results for hydrogen bond potential showed angle dependency of hỵdrogen bond .

For diprotonated states the relative energy difference is only few $\mathrm{kcal} / \mathrm{mol}$. Unprotonated state model. Model $\mathrm{V}$. is the most unstable state among the models we introduced. In a certain protonated class. the relative stability of a model is strongly dependent on the formation of stable hydrogen bonds of the model. Model III forms two intermediate hydrogen bonds network. two weak hydrogen bonds. whereas Model IV forms only two weak hydrogen bonds. Model I forms three weak hydrogen bonds and Model II forms three weak hy'drogen bonds and a hýdrogen bonds of intermediate strength. Though Model $\mathrm{V}$ forms three weak hydrogen bonds. the repulsion between two acetate ions destabilizes the system. Model III is reproduced by good indexes and is more reliable protonated state in HIV-1 protease-A 74704 complexes by binding energy and relative energ:

The relative stabilities of the models in continuum media with $\varepsilon=4$ and 78.3 were calculated and summarized in Table 2. Model III was the most stable in the continuum
Table 3. The predicted free energy differences of the protonated state in HIV-I protease/inhibitor by free energy perturbation simulations

\begin{tabular}{lc}
\hline Perturbation & $\Delta G(\mathrm{kcal} / \mathrm{mol})$ \\
\hline$\Delta G_{1}(\mathrm{~V} \rightarrow \mathrm{III})$ & -58.01 \\
$\Delta G_{2}(\mathrm{~V} \rightarrow \mathrm{II})$ & -53.48 \\
$\Delta G_{3}(\mathrm{III} \rightarrow \mathrm{VIII})$ & 20.14 \\
$\Delta G_{4}(\mathrm{II} \rightarrow \mathrm{III})$ & -4.53 \\
$\Delta G_{5}(\mathrm{II} \rightarrow \mathrm{VIII})$ & 15.61 \\
\hline
\end{tabular}

environment by SCI-PCM calculation in protein core environment $(\varepsilon=4.0)$. In our models, aspartic groups' surface is not exposed to solvent, but many amino acids are near aspartic acid in HIV-I protease, and aspartic groups are a little affected by solvents. SCRF calculations using $\mathrm{SCI}$ PCM were calculated in nonpolar solvent $(\varepsilon=4)$ that was generally represented the character of a nonpolar solvent environment that applied to the environment of the protein core. In the case of HIV-1 protease with A74704. the active site has complex hydrogen bond networks and electrostatic interactions. From our results. the active site is not affected by any high polar solvent medium. the determinations of protonated states in HIV-1 protease with A74704 need the low polarized solvent environment. and suggest that it is very similar to the protein core environment.

To include the influence of the hydrogen bonds between the model and the water and the structural change of the water by the model, molecular dynamic simulations were perfonned with explicit water molecules. In addition to the influence mentioned above. to calculate the relative stabilities between different protonated states, FEP was carried out in conjunction with the MD method. In Table 3. the free energy differences between the protonated states which approach the thermodynamic cycle in Figure 3 are summarized. From the free energy perturbation results. $\Delta G$ presents that Model III is more stable than Model II by $-4.53 \mathrm{kcal} / \mathrm{mol}$, and $\Delta \mathrm{G}_{3}$ suggests that the mutation from monoprotonated state on OD2 of Asp25 to diprotonated state is destabilized bound structure by $20.14 \mathrm{kcal} / \mathrm{mol}$.

The Three FEP simulations for aspartic groups having various protonated states of HIV-1 protease/A74704 complex also reproduced protease structures that agreed well with the crystal structure with an RMS deviation for the protease backbone between 0.56 and $0.65 \mathrm{~A}$. Comparison of the average inhibitor geometries was obtained from each MD simulation with the backbone inhibitor from crystal structure. The residual RMS deviation of the inhibitor backbone atoms is less than $0.65 \mathrm{~A}$ and all comparison within the $\mathrm{Pl}$ and $\mathrm{Pl}$ ' sites of the inhibitor are less than $0.49 \mathrm{~A}$. The geometric results are tabulated between average structure and X-ray cry'stal structure using superimposition in Table 4 , however structural data give no good description of different protonated states

In this study: $\Delta \Delta G$ play's an important role in determining the protonated state. The stability of the protease inhibitor complex corresponds to the difference of protonated states. 
Table 4 . RMS deviations $(A)$ for initial x-ray crvstal structure with average structures" of HVV-1 protease/nulhibitor complexes

\begin{tabular}{lccc}
\hline & $\mathrm{V} \rightarrow$ II & $\mathrm{V} \rightarrow$ III & II $\rightarrow$ VIII \\
\hline Backbone & 0.56 & 0.55 & 0.65 \\
(D25, D125, G27, G127) & 1.07 & 0.93 & 1.15 \\
D25, D125 & 1.10 & 0.90 & 1.03 \\
Inhibitor & 3.46 & 3.48 & 3.47 \\
P1, P1 & 0.46 & 0.49 & 0.47 \\
\hline
\end{tabular}

"Averaged structures were taken from 30.0 ps to $100.0 \mathrm{ps}$.

The results. protonated state calculations using free energy' perturbation. suggest very reliable active site conformation that only OD2 of Asp25 is protonated. Since the energies of OD1 of Asp 125 are different from OD2 of Asp 25 by 4.61 $\mathrm{kcal} / \mathrm{mol}$. our assumption is close to the experimental results by Hyland. ${ }^{11.12}$

Our results are very consistent between $a b$ initio SCRF $(\varepsilon=4)$ and free energy perturbations by molecular mechanics: $\Delta G_{4}$ is $-4.53 \mathrm{kcal} / \mathrm{mol}$ between Model III and Model II. and relative energy by ab initio SCRF $(\varepsilon=4)$ is $-1.21 \mathrm{kcal} / \mathrm{mol}$. $\Delta G_{1}$ is $-58.01 \mathrm{kcal} / \mathrm{mol}$ between Model III and Model V and relative energy by ab initio SCRF $(\varepsilon=4)$ is $32.24 \mathrm{kcal} / \mathrm{mol}$. $\Delta G_{\hat{3}}$ is $-20.14 \mathrm{kcal} / \mathrm{mol}$ between Model III and Model VIII. and relative energy by ab initio SCRF $(\varepsilon=4)$ is $-9.45 \mathrm{kcal} /$ mol. Also the NMR experiments prove that our results are more reliable. Now we proposed the reliable protonated state that the proton should be on the OD2 of Asp25 $\left(\Delta G_{4}=-4.53\right.$ $\mathrm{kcal} / \mathrm{mol}=-1.21 \mathrm{kcal} / \mathrm{mol}$ ) when the HIV-1 protease binds the A74704 inhibitor. Recently. Wang et al ${ }^{26}$ reported chemical shifts and NMR study of the two aspartic acids in HIV-1 protease/KNI 272 complex. which provided the first directly experimental measurement of $\mathrm{p} K_{\mathrm{a}}$ values as protonated state of all aspartic acids. The results clearly show that the protonated state of Asp25 and Aspl25 are independent of $\mathrm{p} K_{\mathrm{a}}$ in the range from 2.5 to 6.2. the OD2 of Asp25 is protonated by $\mathrm{p} K_{\mathrm{a}}=$ over 6.2 while Asp 125 is deprotonated by $\mathrm{p} K_{\mathrm{a}}=2.5$. Baldwin et $a .^{27}$ also reported that semiempirical calculations applied to heavy atom coordinates of the cry'stal structure have predicted that the Asp25 side chain was protonated whereas the Asp125 was deprotonated. which were determined by relative energies. Wonn ${ }^{2.2 .2}$ reported the theoretically very similar results of four possible protonated states of the aspartic acid of HIV-l protease/hydroxyethylene inhibitor by free energy simulations. The free energy perturbation simulation results indicate reasonable protonated state is the OD2 of Asp 25 protonated state. The experimental free energy' difference is $-1.92 \mathrm{kcal} / \mathrm{mol}$. the predicted free energy difference of the HIV-1 protease with hydroxyethylene inhibitor is $\Delta \Delta G=-1.79 \mathrm{kcal} / \mathrm{mol}$ by the free energy simulations.

Harte and Beveridge ${ }^{l+}$ reported the results of unconstrained molecular dynamics simulation of the HIV-l proteaseU85548E complex and MVT-101 complex, where the two catalytic aspartic residues were treated in three different ways: both negative charged. one charged and another neutral and both neutral. However they didn't consider the position of type oxygen (OD1 or OD2). They found that both aspartic residues were neutral, that two aspartic acids were protonated with U85548E inhibitor complex. the complex with inhibitor displaying the lowest RMS deviation from the initial X-ray structure $0.8 \mathrm{~A}$ for backbone and 1.3 A for P1.P1'. MVT-101 inhibitor contains a secondary amine. which will carry a positive charge. thus creating a different environment than the neutral charge U85548E. In the MVT-101 inhibitor and the HIV-1 protease complex. only the dianionic model accurately reproduces the experimentally observed inhibitor geometry. $0.5 \mathrm{~A}$ for backbone and $0.8 \mathrm{~A}$ for P1.P1'

Our calculations can be applied to neutral inhibitor-HIV-1 protease complex. the protonated state is not diprotonated state but monoprotonated state in active site two aspartic acids with neutral inhibitors.

\section{Conclusions}

These results have the potentially significant implications that protonated state of active site in HIV-1 protease A74704 inhibitor complex is monoprotonated state on OD2 of Asp25. This research clearly suggests the protonated state of HIV-1 protease with inhibitor by quantum mechanics and molecular mechanical method. If the inhibitor has neutral character. OD2 of Asp25 must be protonated on. Free energy perturbation has been considered traditionally as a computational technique that directly affords the quantitative estimation of free energy difference between two molecular systems $a b$ initio SCRF calculation is well known that the calculations give correct geometry and energy including the effects of electrons by computational chemistry. Our results give a consistency from the results using ab initio SCRF and molecular mechanics for determining the protonated state of active site in HIV-l protease-inhibitor complex. These calculations provide a theoretical prediction of the protonated states of catalytic aspartic residues with neutral inhibitor complex and may be useful for the evaluation of potential therapeutic targets. We will use mono-protonated state of $\mathrm{OD}_{2}$ of Asp25 to further design the new potent inhibitor.

Acknowledgements. $A b$ initio calculation was performed on a SP2 IBM super computer at Cornell Theory Center. Cray YMP C90 at KISTI in Korea. Free energy' perturbations were performed on a Silicon Graphics Indigo/2 R8000 at Chong Kun Dang Research Institute. This work was supported by Korea Research Foundation Grant. (KRF-2001D00014. KRF-2001-D00015)

\section{References}

1. Mitsuya H.: Yarchoan. R: Broder, S. Science 1990. 2+9. 15331544.

2. Debouck. C. Aids Rearch and Homan Retowinuses 1992. 8. 153 164.

3. Kohl, N. E.: Emini. E. A.: Schleif. W. A.: Davis. L. I.: Heimbach, J. C.: Dixon. R. A.: Scolnick, E. M.; Sigal, I. S. Proc. Natl Acad Sci. LS.A. $1988,85,4686-4690$. 
4. Miller M: Schneider J: Sathyanaravana, B. M: Toth, M. V.: Marshall. G. R.: Clawson. L.: Selk. L.: Kent. S. B. H.: Wlodawer. A. Science 1989. 246.1149-1151.

5. Swain. A. L.: Miller. M.: Green. J.: Rich. D. H.: Schneider. J: Kent. S. B. H.: Wlodawer A. Proc. Natl Acad Si. U. S. A. 1990. $87,8850-8809$

6. Taskolski. M: Tomasselli, A. G.: Sawyer T. K.: Staples. D. G.: Heinrikson. R. L.: Schneider. J.: Kent. S. B. H.: Wlodawer. A. Biochemistry 1991. 30.1600-1609.

7. Fitzgerald. P. M. D.: McKeever. B. M.: VanMiddleworth. J. F.: Springer, J. P.: Heimbach. J. C.: Lell C: Herbert. W. K.: Dixon. R. A. F.: Darke. P. L. J. Bio. Chem. 1990. 265, 14209-14219.

8. Erikson. J.: Neidhart. D. I.: VanDrie, J: Kempf. D. J.: Wang, X. C.: Norbeck. D. W.: Plattner. J. J.: Rittenhouse. J. W: Turon. M. Widenburg. N.: Kollbrenner. W. E.: Simmer. R: Helfrich. R: Paul. D. A.: Kingge. M. Science 1990. 249. 527-533.

9. James. M. N. G.: Salituro. F. S.: Rich. H. D.: Hofmann. T. Proc. Natl Acad Sci L. S A 1982.79.6137.

10. Schechter. I.: Berger A. Biochent. Biophns. Res. Comm. 1967. $27,157-162$.

11. Hyland. L. J.: Tomaszek. T. A.. Jr.: Roberts. G. D.: Carr. S. A.: Magaard. V. W.: Bryan. H. L.: Fakhoury. S. A.: Moore. M. L.: Minnich. M. D.: Culp. J. S.: DesJarlis. R. L.: Meek. T. D. Biochentistry 1991. 30.8441-8453.

12. Hyland, L. J: Tomaszek, T. A.. Ir: Meek, T. D. Biochemistin $199130,8454-8463$.

13. Rodriguez. E. J.: Angeles. T. S.: Meek. T. D. Biochemishy 1993. 32. $12380-12385$.

14. Harte. W. E.. Ir.: Beverage D. L. J. Am. Chem. Soc. 1993. 115. 3883-3886.

15. Chen X: Tropsha. A. J. Afed Chem. 1995, 38. 42-48

16. Ferguson. D. M.: Radmer. R. I.: Kollman. P. A. J. Med Chem. 1991. 34. 2564-2569

17. Frisch. M. J.: Trucks. G. W: Schlegel. H. B.: Gill. P. M. W:
Johnson. B. G.; Robb, M. A.: Cheeseman, J. R.: Keith. T. A.: Petersson. G. A.: Montgomery: J. A.; Raghavachari. K.: AlLaham. M. A.: Zakizewski. V. G.: Ottiz. J. V: Foresman. T. B.: Peng. C. Y.: Ayala. P. A.: Wong. M. W.: Andres. T. L.: Replogle. E. S.: Gomperts. R.: Martin. R. L.: Fox. D. T.: Binkley. T. S.: Defrees. D. J.; Baker. J.; Stewart, J. P.; Head-Gordon. M.; Gonzalez. C.: and Pople, J. A. Gonssian 9f Rerision D.3); Gaussian. Inc.: Pittsburgh. PA. 1995.

18. Keith. T. A.: Foresmant. T. B.: Frisch. M. T.: Weiberg. K. B. manuscript in preparation.

19. Foresman. T. B.: Keith. T. A.: Weiberg. K. B.: Frisch. M. J. $J$. Pho Chent 1996, 100. 16098-16104.

20. Mezei. M. J. Chent Phs 1987.86.7084-7088

21. Molecular dynamic simulation was performed using the Insighth Discover (2.9.5). MSI Inc: Insight! (950):Discover 2.9 .5 Molecular Modeling Sottware: San Diego. CA. 1995.

22. Hagler. A. I.: Lifson. S.: Dauber. P. J. Am. Chem. Soc 1979. 101 $5122-5130$

23. Lifson. S.; Hagler. A. T.: Dauber. P. J. Ant. Chem. Soc. 1979. 101. 5131.

24. Fixed geometry were used of HIV-1 protease in which fised amino acid residues were listed. 1-7. 11-21. 34-46. 55-75. 89-99. $101-107.111-121.134-146.155-175$ and $189-199$.

25. No. K. T.; Kwon, O. Y.: Kim. S. Y.; Jhon. M. S.: Scheraga, A. H. $J$. Phus Chent 1995, 99.3478.

26. Wang, Y.-X: Freedberg, I. D.: Yamazaki. T; Wingfield. T. P.; Stahl. J. S.: Kautman. D. T.: Kiso. Y.: Torchia. A. D. Biochemisty 1996. $31.9945-9950$.

27. Baldwin. E. T.: Bhat. T. N.: Gulnik. S.: Liu. B.: Topol. I. A.: Kiso. Y.: Mimoto, T.: Mitsuya. H.: Erickson. J. W. Sintcture 1995. 3. $581-590$.

28. Won, Y. Bull Korem Chem. Soc. 2000. 21. 1207-1212.

29. Won. H.: Kim. J. R.: Ko. K.: Won. Y. Bull. Korean Chem. Soc. 2003. $23.27-28$. 